\title{
Impact of a care pathway for COPD on adherence to guidelines and hospital readmission: a cluster randomized trial
}

This article was published in the following Dove Press journal:

International Journal of COPD

23 November 2016

Number of times this article has been viewed

\author{
Kris Vanhaecht ${ }^{1,2}$ \\ Cathy Lodewijckx' \\ Walter Sermeus' \\ Marc Decramer ${ }^{3,4}$ \\ Svin Deneckere ${ }^{1,5}$ \\ Fabrizio Leigheb ${ }^{6}$ \\ Paulo Boto ${ }^{7}$ \\ Seval Kul ${ }^{8}$ \\ Deborah Seys ${ }^{\prime}$ \\ Massimiliano Panella ${ }^{1,6}$ \\ 'Department of Public Health and \\ Primary Care, Leuven Institute for \\ Healthcare Policy, KU Leuven - \\ University of Leuven, ${ }^{2}$ Department \\ of Quality Management, University \\ Hospitals Leuven, ${ }^{3}$ Department of \\ Clinical and Experimental Medicine, \\ KU Leuven - University of Leuven, \\ ${ }^{4}$ University Hospitals Leuven, \\ Leuven, ${ }^{5}$ Medical Department, \\ Delta Hospitals Roeselare, \\ Roeselare, Belgium; 'Department of \\ Translational Medicine, University \\ of Eastern Piedmont, Vercelli, Italy; \\ ${ }^{7}$ Department of Health Services \\ Policy and Management, Centro \\ de Investigação em Saúde Pública, \\ Escola Nacional de Saúde Pública, \\ Universidade Nova de Lisboa, Lisbon, \\ Portugal; ${ }^{8}$ Department of Biostatistics, \\ School of Medicine, University of \\ Gaziantep, Gaziantep, Turkey
}

Correspondence: Kris Vanhaecht Department of Public Health and Primary Care, Leuven Institute for Healthcare Policy, KU Leuven - University of Leuven, Kapucijnenvoer 35/3, 3000 Leuven, Belgium

Tel +32 I637 7760

Fax +3216336970

Email kris.vanhaecht@kuleuven.be
Purpose: Current in-hospital management of exacerbations of COPD is suboptimal, and patient outcomes are poor. The primary aim of this study was to evaluate whether implementation of a care pathway (CP) for COPD improves the 6 months readmission rate. Secondary outcomes were the 30 days readmission rate, mortality, length of stay and adherence to guidelines.

Patients and methods: An international cluster randomized controlled trial was performed in Belgium, Italy and Portugal. General hospitals were randomly assigned to an intervention group where a CP was implemented or a control group where usual care was provided. The targeted population included patients with COPD exacerbation.

Results: Twenty-two hospitals were included, whereof 11 hospitals ( $\mathrm{n}=174$ patients) were randomized to the intervention group and 11 hospitals ( $\mathrm{n}=168$ patients) to the control group. The CP had no impact on the 6 months readmission rate. However, the 30 days readmission rate was significantly lower in the intervention group $(9.7 \% ; 15 / 155)$ compared to the control group $(15.3 \% ; 22 / 144)$ (odds ratio $=0.427 ; 95 \%$ confidence interval $0.222-0.822 ; P=0.040$ ). Performance on process indicators was significantly higher in the intervention group for 2 of 24 main indicators $(8.3 \%)$.

Conclusion: The implementation of this in-hospital CP for COPD exacerbation has no impact on the 6 months readmission rate, but it significantly reduces the 30 days readmission rate.

Keywords: COPD, care pathway, readmission, quality improvement, cluster randomized controlled trial

\section{Introduction}

Exacerbations of COPD are a leading cause of hospital admissions worldwide. Thirty-five percent of COPD patients have at least 1 admission a year, with up to $30 \%$ readmitted within the 6 months after discharge. ${ }^{1-3}$ Adequate in-hospital management is expected to reduce readmission rates. ${ }^{4}$ Although several worldwide established guidelines are available for the management of COPD, 2,5 the current in-hospital management of COPD exacerbations is suboptimal, and outcomes with regard to readmission and mortality are poor. ${ }^{6,7}$

Care pathways (CPs) are widely used for optimizing adherence to guidelines and improving outcomes. ${ }^{8-10}$ They are defined as "a complex intervention for the mutual decision making and organization of predictable care for a well-defined group of patients during a well-defined period". ${ }^{11}$ Although COPD exacerbations are well suited to be treated in CPs, existing research on effectiveness is limited. Moreover, not one randomized study on COPD CPs has been reported up to date. ${ }^{12-15}$ As CPs are complex interventions that induce change at different levels of the organization, 
cluster randomized controlled trials (CRCTs) should be used to study their impact. ${ }^{16}$

This trial is a CRCT on CP effectiveness launched by the European Pathway Association (E-P-A) in 2009. ${ }^{11}$ The primary aim of this study was to evaluate whether implementation of a CP improves the 6 months readmission rate for patients with a COPD exacerbation. Secondary outcomes were the 30 days readmission rate, mortality, length of stay and adherence to guidelines.

\section{Patients and methods}

\section{Study design and participants}

A pragmatic $\mathrm{CRCT}^{17}$ was conducted, and the clusters included general hospitals out of Belgium, Ireland, Italy and Portugal, where patients hospitalized for a COPD exacerbation were cared for by a multidisciplinary team. Hospitals were randomized to either an intervention group, where a CP was developed and implemented, or a control group, where usual care was provided (Figure 1). Usual care means that team members provide the same care during the study period as they were doing before implementation in the study. The study was registered as a CRCT at ClinicalTrials.gov (identifier: NCT00962468). Ethical approval was obtained on 3 levels. First, ethical approval was sought by the ethical committee of the research center at the country level. This included approval by the ethical committee of the coordinating center at Leuven University (identifier: ML5617), the National Committee of Data Protection for Portugal (6497/2011) and the ethical committee of the AOU Maggiore della Carità di Novara for Italy (625,21/07/2011). Second, ethical approval was sought with regard to participation in the trial at the cluster level by the ethical committee of each of the participating hospitals (Table S1). Finally, individual written informed consent was obtained from all patients with regard to participation in the study and access to the patient record.
General hospitals could develop a CP when they provided written agreement to participate in the study. When hospitals were randomized into the control group, they agreed to not develop and implement a COPD CP within the time frame of the study. Eligibility criteria for patients were 1) hospital admission with COPD exacerbation as the primary diagnosis, 2) hospitalized for at least 48 hours, 3) admitted in a ward where COPD exacerbations were usually treated, 4) able to understand and read the native language and 5) provision of written informed consent. Patients could be included in the study only once, specifically at their first admission during the study period. Patients were excluded 1) if already included in another study of which the measurements could influence the measurements or outcomes of this study or 2) if they needed invasive positive pressure ventilation at admission to the hospital. ${ }^{11}$

Enrollment of hospitals was done by the E-P-A, in close collaboration with the national E-P-A coordinator of each country. After consent, a study coordinator was appointed in each participating hospital. ${ }^{11}$

\section{Randomization and masking}

Allocation concealment at team level was not possible and therefore general hospitals were stratified by country level, hospital type (teaching versus non-teaching), hospital size ( $<600$ and $\geq 600$ beds) and annual patient volume for COPD exacerbation $(<300 \text { patients and } \geq 300 \text { patients })^{18,19}$ and then randomly assigned to the intervention group or to the control group. The allocation sequence was computer generated by a principal investigator at the coordination center at Leuven University, using a random number list and statistical software (http://www.randomizer.org). Study coordinators and teams were informed on their allocation after randomization. To minimize the risk for testing bias, the detailed data collection protocol was sent to the study

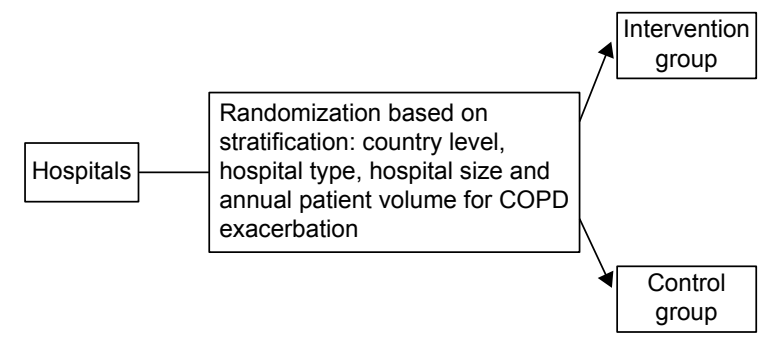

Intervention:

1) Evaluation on the quality and organization of care before CP implementation ${ }^{11}$

2) Providing a set of identified evidencebased key-interventions ${ }^{20}$ on in-hospital management of COPD exacerbations to each multidisciplinary team

3) Training of pathway facilitators on $C P$ development and implementation by using the 7-phase protocol based on the Deming-PDSA cycle ${ }^{21}$
Measurement:

Clinical and team audit:

- Process indicators

- Outcome indicators

- Team indicators

Measurement:

Clinical and team audit:

- Process indicators

- Outcome indicators

- Team indicators

Figure I Study overview.

Abbreviation: CP, care pathway. 
coordinator of each hospital just before the start of the data collection. Furthermore, a logbook was kept for included patients in order to be able to connect the patient identity to the study number. In addition, to check for selection bias, for each patient admitted with COPD exacerbation during the recruitment period but excluded, baseline characteristics and the reason for exclusion were reported in a logbook for excluded patients.

Finally, in order to prevent assessment bias, all data, except for the measurements at discharge, were collected by an external researcher outside the clinical team. At discharge, a structured interview of the patient by a team member was performed to collect information on previous home situation and therapy before admission, which did not imply any risk for bias of the measurements.

\section{Intervention}

In the intervention group, a CP was implemented at hospital-level $\mathrm{CP}$, and the intervention was composed of 3 active components (Figure 1). ${ }^{11} 1$ ) An evaluation on the quality and organization of care before $\mathrm{CP}$ implementation. In every hospital, a clinical audit was performed during a 2- to 3-month period, 6 months before developing the $\mathrm{CP}$. Hospitals received a feedback report describing their performance compared to evidence-based guidelines and the performance of all other participating hospitals. The purpose of this feedback report was to help the hospitals in understanding the deficiencies in their actual organization of the care. This feedback report was discussed during a workshop, held before the start of the CP development. This workshop, as part of the intervention, was held before the start of the CP development and was attended by pneumologist, (head) nurse, physiotherapists and/or pathway facilitator of each participating hospital. During this workshop, the purpose of the EQCP study was presented, and the feedback report and the key interventions were discussed. This workshop closed with the next steps of the study, and time plans were presented. 2) Providing a set of identified evidence-based key interventions ${ }^{20}$ on in-hospital management of COPD exacerbations to each multidisciplinary team. The set of key interventions were based on literature, an international Delphi study and consensus meeting with multidisciplinary expert panel. ${ }^{20}$ During the workshop, described earlier, these key interventions were presented and discussed. During the implementation phase, teaching sessions were organized by a pneumologist and a respiratory clinical nurse specialist concerning those key interventions for which teams experienced implementation difficulties, ie, administration of corticoid therapy, education on self-management strategies and inhalation therapy. Finally, slide kits were provided to the pathway facilitator of each hospital, ie, regarding administration of oxygen therapy, in order to support the facilitators in the education of their team members regarding the different key interventions. 3) Training of pathway facilitators on CP development and implementation by using the 7-phase protocol based on the Deming-Plan Do Study Act cycle. ${ }^{21}$ For the development and implementation of the CP, the findings of the evaluation of the care process and the set of evidence-based key interventions were used. Meetings with the pathway facilitators were organized to further discuss the feedback reports and to address problems in implementation. Furthermore, a change expert supported change and exchange of knowledge and best practices. ${ }^{11,22}$ The teams developed a CP over a 6- to 8-month period. ${ }^{23}$ In the control group, patients received the usual care, and no intervention was developed or implemented before.

\section{Measurement}

The primary outcome was the 6 months readmission rate. Secondary outcomes included the 30 days readmission rate, the 30 days and the 6 months mortality rate, length of stay and results on 24 main process indicators, categorized in diagnostic, pharmacological and non-pharmacological management, respectively. Twelve of 24 main process indicators were built of 2 or more subcomponents. For the 30 days and the 6 months readmission rate, which refers to COPD-specific readmission, only patients alive at, respectively, the 30 days and the 6 months were included in the analyses. ${ }^{20}$ Additionally, demographic and COPD-specific data were collected.

The measurement period started 2-3 months after the end of the implementation period. ${ }^{21}$ Data were collected by structured interviews performed by a team member at discharge, patient questionnaires completed at discharge and 30 days after discharge, structured telephone interviews at 30 days and 6 months after discharge performed by the study coordinator and a patient record analysis after discharge of the patient, performed by the study coordinator, together with a clinician outside the care team. In each hospital, all data were collected centrally and subsequently provided to the national coordinators of all participating countries. Data input was performed in a central database at Leuven University and guided by using a rigorous data input protocol.

\section{Statistical analysis}

Sample size calculation was performed according to standard criteria for CRCTs. ${ }^{11,24}$ Based on a number of 20 consecutive admitted patients in each unit, 20 hospitals needed to be included in both the intervention and the control groups. 
Briefly, the intracluster correlation coefficient (ICC) for the 6 months readmission rate was estimated to be equal to 0.018 , leading to a design effect of 1.342 . Using standard $0.05 \alpha$ error and assuming a reduction of $11 \%$ in the 6 months readmission rate (from $41 \%$ to $30 \%$ ), ${ }^{25,26}$ a sample size of 398 patients per arm was required to obtain a statistical power of $0.80 .{ }^{11}$

A univariate analysis was carried out testing the baseline characteristics between intervention and control groups by using the chi-square test, Mann-Whitney $U$-test and independent sample $t$-test for categorical, ordinal and continuous variables, respectively. The process indicators were analyzed by 2-level mixed-effects logistic regression model, accounting for the clustering effect. The outcome indicators are analyzed by using a 2-level mixed-effects logistic and linear regression model for categorical and continuous variables, respectively, accounting for the clustering effect. For the adjusted outcomes, the significant variables $(P<0.1)$, as determined by the univariate analysis and the intervention, were included in the final model. Multicollineairity was assessed.

Statistical significance was defined as a 2 -sided $P$-value of 0.05 . All analyses were intention to treat, performed by using R package lme4 (version 3.1.0) and MPlus 7.3 for ICC calculations.

\section{Results}

Initially, 65 hospitals were eligible for inclusion. After receiving the detailed study protocol, 22 hospitals decided to participate in the study. Of the 43 hospitals who did not take part in the study, 26 hospitals decided to not participate because they found the workload associated with the study too high. Furthermore, all 14 Irish hospitals dropped out because of reorganization of the Irish health care system and 3 hospitals dropped out because of internal reorganization.

Regardless of the drop-out, by chance 11 hospitals were allocated to the intervention group and 11 hospitals to the control group. In total, 342 patients, 174 in the intervention group and 168 in the control group, were recruited. The Belgian hospitals included patients between October 2010 and November 2011, while the Italian and Portuguese hospitals included patients between January 2013 and April 2014. In the intervention group, respectively, 10 patients and 1 patient were lost to follow-up at 30 days and 6 months after discharge, because of not reachable by the study coordinator. In the control group, respectively, 7 and 13 patients were lost to follow-up at 30 days and 6 months after discharge for the same reason (Figure 2).

With regard to patient characteristics, the groups were highly comparable except for COPD severity at admission (higher in control group, $P=0.018$ ), diabetes (higher in the control group, $P=0.007$ ) and low body mass index (more present in the intervention group, $P=0.003$ ). Also, although not significant, cardiac failure and hospitalization in the year before index admission were higher in the control group. At cluster level, both groups were comparable for type, size and annual volume of patients admitted with COPD exacerbation (Table 1).

The 6 months readmission rate was lower in the intervention group (27.3\%) compared to the control group (33.0\%), though this result was not found statistically significant (adjusted odds ratio [OR] $=0.642,95 \%$ CI $0.347-1.188$, $P=0.158)$. Readmission rate at 30 days was statistically significantly lower $(9.7 \%)$ in the intervention group compared to the control group (15.3\%) (adjusted OR $=0.427,95 \%$ CI 0.222-0.822) (Tables 2 and 3). No significant differences were found for the 30 days and the 6 months mortality rate and length of stay.

Results on the individual process indicators are presented in Table 4. Performance on the individual process indicators was significantly higher for only 2 of 24 main indicators (8.3\%) and for 9 of 41 subcomponents (22.0\%). The largest differences were determined for the main indicators regarding non-pharmacological management (range of improvements: $0.7-45.9$ percentage points). The mean adherence to the total of 24 measured process indicators was 59.4\% (range: $18.8 \%-94.7 \%$ ) in the intervention group and $49.4 \%$ (range: $11.8 \%-88.2 \%)$ in the control group $(P=0.071)$. No patient received all the care they should receive.

\section{Discussion}

The implementation of this CP has no significant effect on the 6 months readmission rate. However, the 30 days readmission rate was significantly lower in the intervention group (9.7\%) compared to the control group (15.3\%). Performance on process indicators was significantly higher in the intervention group for 2 of 24 main indicators $(8.3 \%)$ and for 9 of 41 subcomponents $(22.0 \%)$.

Before the launch of this study, only 5 national CRCTs on CPs had been conducted. ${ }^{23,27-30}$ This first international trial provides new knowledge on the design of a multicountry CRCT. In comparison to former pathway studies, 1) the impact of the $\mathrm{CP}$ on the care itself was comprehensively investigated, while earlier studies focused primarily on outcomes, ${ }^{12-15}$ 2) training of teams was an active component of the intervention and 3) a clinical audit, as part of the intervention, allowed each hospital to focus on those key interventions that showed most room for improvement. ${ }^{21}$ 


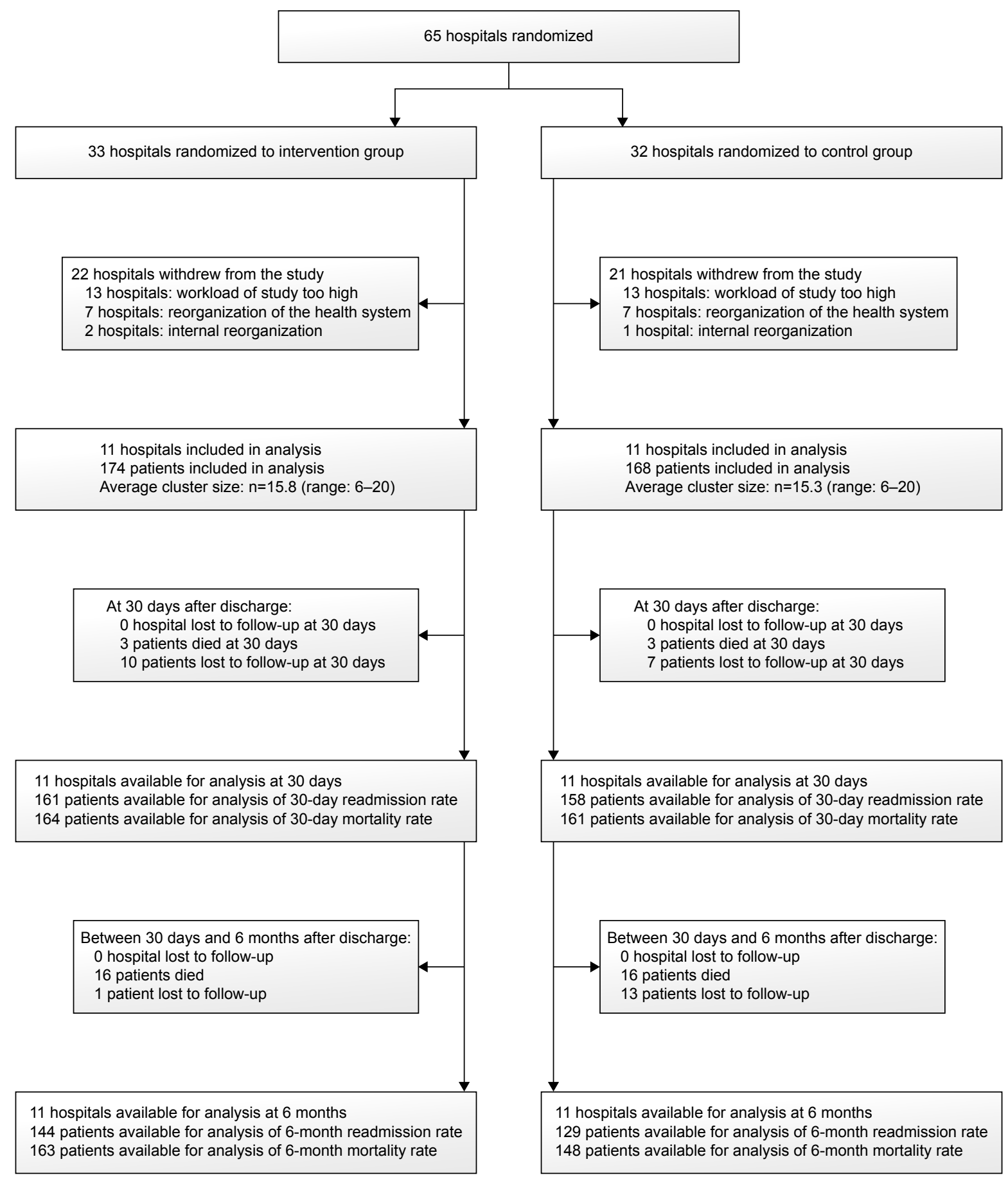

Figure 2 Participant flow at hospital and patient level.

A weakness of the study is that 43 hospitals withdrew after randomization, which resulted in a smaller sample size than initially targeted. It is reasonable to assume that the lower power of the study may have led to a failure in detecting statistically significant differences. The experience in this study is that a multicountry CRCT poses a major challenge due to standardization of the intervention in order to deliver the "same" intervention at the different sites, logistic, economic and cultural issues. These conditions should be considered carefully before starting an international CRCT. The study was underpowered, but it was decided not to increase the number of patients within the clusters because increasing the sample size per cluster does not increase power. ${ }^{31}$

Research on COPD CPs is very limited. Previous studies described positive effects on blood sampling, daily weight measurement, arterial blood gas measurement, referral to 
Table I Baseline characteristics of patients and hospitals

\begin{tabular}{|c|c|c|c|}
\hline & $\begin{array}{l}\text { Intervention group } \\
(\mathrm{n}=174)\end{array}$ & $\begin{array}{l}\text { Control group } \\
(n=168)\end{array}$ & $P$-value ${ }^{a}$ \\
\hline \multicolumn{4}{|l|}{ Individual level } \\
\hline Age (years) & $69.4(10.8)$ & $70.4(9.7)$ & 0.337 \\
\hline Gender & & & 0.645 \\
\hline Men & II $6 / 174$ (66.7) & II6/I68 (69.0) & \\
\hline Women & $58 / 174(33.3)$ & $52 / 168(31.0)$ & \\
\hline COPD severity at admission & & & 0.018 \\
\hline GOLD I: mild & $8 / I 7 \mid(4.7)$ & I2/I54 (7.8) & \\
\hline GOLD II: moderate & $55 / I 7 \mid(32.2)$ & $29 / 154(18.8)$ & \\
\hline GOLD III: severe & 68/I7I (39.8) & $57 / / 54(37.0)$ & \\
\hline GOLD IV: very severe & $40 / 171(23.4)$ & $56 / 154(36.4)$ & \\
\hline Unknown & 3 & 14 & \\
\hline Smoking status & & & 0.879 \\
\hline No smoker & $11 / 17 \mid(6.4)$ & $13 / 168(7.7)$ & \\
\hline Ex-smoker & $87 / 17 \mid(50.9)$ & $86 / 168(51.2)$ & \\
\hline Active smoker & 73/I7I (42.7) & $69 / 168$ (4I.I) & \\
\hline Unknown & 3 & 0 & \\
\hline Hospitalization in the year & & & 0.234 \\
\hline \multicolumn{4}{|l|}{ before index admission ${ }^{\mathrm{b}}$} \\
\hline 0 & $101 / 154(65.6)$ & $85 / 145(58.6)$ & \\
\hline$\geq 1$ & $53 / 154(34.4)$ & $60 / 145(41.4)$ & \\
\hline Unknown & 20 & 23 & \\
\hline Charlson comorbidity index & & & 0.160 \\
\hline$\leq 2$ & $59 / 174(33.9)$ & $45 / 168(26.8)$ & \\
\hline$>2$ & $115 / 174(66.1)$ & $123 / 168(73.2)$ & \\
\hline Cardiac failure ${ }^{c}$ & $56 / 17 \mid(32.7)$ & $69 / 165(41.8)$ & 0.091 \\
\hline Unknown & 3 & 3 & \\
\hline Diabetes $^{d}$ & $21 / 17 \mid(12.3)$ & $39 / 165(23.6)$ & 0.007 \\
\hline Unknown & 3 & 3 & \\
\hline \multicolumn{4}{|l|}{ BMI } \\
\hline$<20$ & $46 / 159(28.9)$ & $23 / 156(14.7)$ & \\
\hline$\geq 20$ & II3/I59 (7I.I) & I33/I56 (85.3) & 0.003 \\
\hline Unknown & 15 & 12 & \\
\hline \multicolumn{4}{|l|}{ Hospital level } \\
\hline Teaching hospitals & $5 / 11(45.5)$ & $5 / 11(45.5)$ & 1.000 \\
\hline$>600$ beds & $2 / 11(18.2)$ & $3 / 11(27.3)$ & 0.610 \\
\hline$>300$ patients admitted with & $2 / 11(18.2)$ & I/II (9.I) & 0.531 \\
\hline COPD exacerbation per year & & & \\
\hline
\end{tabular}

Notes: Data are mean (SD) or n/N (\%). ${ }^{a}$ Chi-square test, Mann-Whitney U-test and independent sample $t$-test for categorical, ordinal and continuous variables, respectively. 'Index admission: first admission during the study, at point of inclusion. Includes heart failure, arrhythmia, valvular disease, acute myocardial infarction and ischemic heart disease. Includes diabetes uncomplicated and complicated.

Abbreviations: BMI, body mass index; GOLD, Global Initiative for Obstructive Lung Disease; SD, standard deviation.

Table 2 Results on outcome indicators

\begin{tabular}{|c|c|c|c|c|c|c|c|}
\hline & \multirow{2}{*}{$\begin{array}{l}\text { Intervention } \\
\text { group }\end{array}$} & \multirow{2}{*}{$\begin{array}{l}\text { Control } \\
\text { group }\end{array}$} & \multicolumn{3}{|c|}{ Difference unadjusted } & \multicolumn{2}{|l|}{ Difference adjusted } \\
\hline & & & OR (95\% CI) & $P$-value & ICC & OR $(95 \% \mathrm{Cl})$ & $P$-value \\
\hline 6 months readmission & $38 / 139(27.3)$ & $38 / 115(33.0)$ & $0.817(0.454-1.470)$ & 0.500 & 0.015 & $0.642(0.347-1.188)$ & 0.158 \\
\hline 30 days readmission & $15 / 155(9.7)$ & $22 / 144(15.3)$ & $0.570(0.223-1.450)$ & 0.241 & 0.041 & $0.427(0.222-0.822)$ & 0.040 \\
\hline 6 months mortality & $18 / 157(|1| .5)$ & $18 / 134(13.4)$ & $0.822(0.3 \mid 4-2.157)$ & 0.691 & 0.065 & $0.611(0.189-1.972)$ & 0.410 \\
\hline 30 days mortality & $3 / 168(1.8)$ & $2 / 153(1.3)$ & $0.965(0.192-4.840)$ & 0.965 & NC & $0.880(0.126-6.166)$ & 0.898 \\
\hline Length of stay & $12.0(10.5)$ & $12.8(12.4)$ & $0.550(0.02|-| 4.284)$ & 0.720 & 0.043 & $0.901(0.015-53.049)$ & 0.960 \\
\hline
\end{tabular}

Notes: Adjusted for COPD severity at admission, cardiac failure, diabetes, BMI and intervention-control group.

Abbreviations: BMI, body mass index; $95 \% \mathrm{Cl}, 95 \%$ confidence interval; ICC, intracluster correlation coefficient; NC, not possible to calculate; OR, odds ratio. 
Table 3 Two-level mixed-effects regression model

\begin{tabular}{|c|c|c|c|c|}
\hline & \multicolumn{2}{|c|}{6 months readmission } & \multicolumn{2}{|l|}{30 days readmission } \\
\hline & OR $(95 \% \mathrm{Cl})$ & $P$-value & OR $(95 \% \mathrm{CI})$ & $P$-value \\
\hline \multicolumn{5}{|l|}{ COPD severity at admission } \\
\hline GOLD I & I.0 (Reference) & - & I.0 (Reference) & - \\
\hline GOLD II & $0.652(0.155-2.746)$ & 0.560 & $0.304(0.067-1.394)$ & 0.252 \\
\hline GOLD III & $0.724(0.183-2.867)$ & 0.645 & $0.220(0.05 I-0.94 I)$ & $0.04 I$ \\
\hline GOLD IV & $0.834(0.204-3.408)$ & 0.801 & $0.337(0.079-1.436)$ & 0.141 \\
\hline Cardiac failure & $1.236(0.655-2.333)$ & 0.513 & $1.149(0.5 \mathrm{II}-2.580)$ & 0.737 \\
\hline Diabetes & $0.945(0.395-2.259)$ & 0.898 & $1.508(0.540-4.210)$ & 0.433 \\
\hline BMI & $0.7 \mid 4(0.363-1.405)$ & 0.300 & $0.697(0.289-1.686)$ & 0.424 \\
\hline $\begin{array}{l}\text { Main effect adjusted for } \\
\text { (intervention-control group) }\end{array}$ & $0.642(0.347-1.188)$ & 0.158 & $0.427(0.222-0.822)$ & 0.040 \\
\hline
\end{tabular}

Abbreviations: BMI, body mass index; $95 \% \mathrm{Cl}$, 95\% confidence interval; GOLD, Global Initiative for Chronic Obstructive Lung Disease; OR, odds ratio.

Table 4 Results on process indicators: main level and subcomponent level ${ }^{\mathrm{a}}$

\begin{tabular}{|c|c|c|c|c|}
\hline & $\begin{array}{l}\text { Intervention group, } \\
\mathrm{n} / \mathrm{N}(\%)\end{array}$ & $\begin{array}{l}\text { Control group, } \\
\mathrm{n} / \mathrm{N}(\%)\end{array}$ & $P$-value & $\begin{array}{l}\text { Difference between } \\
\text { intervention and } \\
\text { control group }\end{array}$ \\
\hline \multicolumn{5}{|l|}{ Process indicators on diagnostic management } \\
\hline I. Performance of $A B G$ measurement during first 24 hours of admission & I36/174 (78.2) & I26/I68 (75.0) & 0.526 & 3.2 \\
\hline 2. Performance of chest $X$-ray during first 24 hours of admission & $160 / 174(92.0)$ & 143/I $68(85.1)$ & 0.773 & 6.9 \\
\hline a. Performance of chest $\mathrm{X}$-ray during hospitalization & I72/174 (98.9) & $164 / 168(97.6)$ & 0.445 & 1.3 \\
\hline b. Performance occurred within first 24 hours of admission & $160 / 172(93.0)$ & I43/I64 (87.2) & 0.895 & 5.8 \\
\hline 3. Performance of electrocardiogram during first 24 hours of admission & I34/I74 (77.0) & $121 / 168(72.0)$ & 0.847 & 5.0 \\
\hline a. Performance of electrocardiogram during hospitalization & $161 / 174(92.5)$ & I49/168 (88.7) & 0.442 & 3.8 \\
\hline b. Performance occurred within first 24 hours of admission & $134 / 161(83.2)$ & $121 / 149(81.2)$ & 0.867 & 2.0 \\
\hline $\begin{array}{l}\text { 4. Sputum culture and antibiogram in patients with purulent sputum } \\
\text { and/or before starting antibiotics }\end{array}$ & $110 / 154(71.4)$ & $88 / 151(58.3)$ & 0.626 & 13.1 \\
\hline 5. Measurement of FEV , during current hospitalization & $123 / 174(70.7)$ & $102 / 168(60.7)$ & 0.248 & 10.0 \\
\hline \multicolumn{5}{|l|}{ Process indicators on pharmacological management } \\
\hline 6. Prescription of short-acting bronchodilators during hospitalization & $160 / 174(92.0)$ & II3/168 (67.3) & 0.116 & 24.7 \\
\hline 7. Prescription of long-acting bronchodilators during hospitalization & 139/174 (79.9) & | 39/| 68 (82.7) & 0.606 & -2.8 \\
\hline 8. Prescription of $30-40 \mathrm{mg}$ of oral prednisolone daily for $7-10$ days & $50 / 174(28.7)$ & $19 / 168(11.3)$ & 0.091 & 17.4 \\
\hline $\begin{array}{l}\text { a. Systemic glucocorticosteroids prescribed during } \\
\text { hospitalization }\end{array}$ & $152 / 174(87.4)$ & $135 / 168(80.4)$ & $<0.001$ & 7.0 \\
\hline $\begin{array}{l}\text { b. Prescription of glucocorticosteroids for } 7 \text { days to maximum } \\
\text { 14 days starting at admission in patients in which glucocorticoids } \\
\text { were prescribed }\end{array}$ & $113 / 152(74.3)$ & $83 / 135(61.5)$ & 0.203 & 12.8 \\
\hline $\begin{array}{l}\text { c. Dose of glucocorticosteroids prescribed during first } 7 \text { days } \\
\text { of hospitalization was } 30-40 \mathrm{mg} \text { in patients in which } \\
\text { glucocorticoids were prescribed }\end{array}$ & $134 / 152(88.2)$ & $93 / 135(68.9)$ & 0.022 & 19.3 \\
\hline $\begin{array}{l}\text { d. Oral administration since hospitalization day } 2 \text { or earlier in } \\
\text { patients in which glucocorticoids were prescribed }\end{array}$ & $80 / 152(52.6)$ & $43 / 135(31.9)$ & 0.312 & 20.7 \\
\hline 9. Prescription of antibiotics & I39/174 (79.9) & I39/168 (82.7) & 0.655 & -2.8 \\
\hline \multicolumn{5}{|l|}{ Process indicators on non-pharmacological management } \\
\hline $\begin{array}{l}\text { 10. Administration of controlled oxygen therapy in patients hypoxemic } \\
\text { during admission }\end{array}$ & $44 / 47(93.6)$ & $39 / 42(92.9)$ & 0.996 & 0.7 \\
\hline II. Assessment of smoking status & $161 / 174(92.5)$ & I53/I68 (9I.I) & 0.970 & 1.4 \\
\hline 12. Smoking cessation intervention in active smokers at admission & $3 / 42(7.1)$ & $0 / 40(0.0)$ & NC & 7.1 \\
\hline $\begin{array}{l}\text { a. Providing of spoken information about quitting strategies } \\
\text { by physician or nurse in active smokers at admission }\end{array}$ & $23 / 42(54.8)$ & $10 / 40(25.0)$ & 0.030 & 29.8 \\
\hline $\begin{array}{l}\text { b. Providing of smoking cessation leaflet in active smokers at } \\
\text { admission }\end{array}$ & I5/42 (35.7) & $1 / 40(2.5)$ & 0.049 & 33.2 \\
\hline
\end{tabular}




\begin{tabular}{|c|c|c|c|c|}
\hline & $\begin{array}{l}\text { Intervention group, } \\
\mathrm{n} / \mathrm{N}(\%)\end{array}$ & $\begin{array}{l}\text { Control group, } \\
\mathrm{n} / \mathrm{N}(\%)\end{array}$ & $P$-value & $\begin{array}{l}\text { Difference between } \\
\text { intervention and } \\
\text { control group }^{\mathrm{a}}\end{array}$ \\
\hline $\begin{array}{l}\text { c. Providing of quitting aids before discharge in active smokers } \\
\text { at admission who wants to quit smoking by using quitting aids } \\
\text { (patches, gum, medication) }\end{array}$ & $9 / 16(56.3)$ & $3 / 9(33.3)$ & 0.277 & 23.0 \\
\hline $\begin{array}{l}\text { d. Referral to counseling in active smokers at admission who wants } \\
\text { to quit smoking by counseling (individual/group) }\end{array}$ & $2 / 3(66.7)$ & $\mathrm{I} / 4(25.0)$ & 0.880 & 41.7 \\
\hline $\begin{array}{l}\text { e. Contacting of patient in the following month after discharge by the } \\
\text { general practitioner, primary nurse or somebody of the hospital } \\
\text { about his/her smoking or attempt to quit in active smokers at } \\
\text { admission }\end{array}$ & $6 / 42(14.3)$ & $4 / 40(10.0)$ & 0.726 & 4.3 \\
\hline $\begin{array}{l}\text { 13. Adequate education regarding inhaler therapy in patients in } \\
\text { which inhaler therapy is prescribed }\end{array}$ & $61 / 173(35.3)$ & I5/165 (9.1) & 0.037 & 26.2 \\
\hline $\begin{array}{l}\text { a. Providing of education regarding inhaler medication in patients in } \\
\text { which inhaler therapy is prescribed }\end{array}$ & $110 / 173(63.6)$ & $33 / 165(20.0)$ & 0.118 & 43.6 \\
\hline $\begin{array}{l}\text { b. Providing of education regarding inhaler technique in } \\
\text { patients in which inhaler therapy is prescribed }\end{array}$ & $114 / 173$ (65.9) & $33 / 165(20.0)$ & 0.049 & 45.9 \\
\hline $\begin{array}{l}\text { c. Providing of education regarding inhaler device in patients in which } \\
\text { inhaler therapy is prescribed }\end{array}$ & $109 / 173(63.0)$ & $37 / 165$ (22.4) & 0.171 & 40.6 \\
\hline $\begin{array}{l}\text { d. Providing of a leaflet with explanation regarding inhaler therapy in } \\
\text { patients in which inhaler therapy is prescribed }\end{array}$ & $8 \mathrm{I} / \mathrm{I} 70(47.6)$ & $53 / 162(32.7)$ & 0.192 & 14.9 \\
\hline $\begin{array}{l}\text { 14. Education regarding home oxygen therapy in patients in which } \\
\text { home oxygen is prescribed }\end{array}$ & $14 / 52(26.9)$ & $0 / 64(0.0)$ & NC & 26.9 \\
\hline $\begin{array}{l}\text { a. Providing of education regarding oxygen source in patients } \\
\text { in which home oxygen is prescribed }\end{array}$ & $25 / 52(48.1)$ & I5/64 (23.4) & 0.374 & 24.7 \\
\hline $\begin{array}{l}\text { b. Providing of education regarding equipment (cannulae/mask) } \\
\text { in patients in which home oxygen is prescribed }\end{array}$ & $24 / 52(46.2)$ & I3/64 (20.3) & 0.414 & 25.9 \\
\hline $\begin{array}{l}\text { c. Providing of education regarding safety precautions in patients } \\
\text { in which home oxygen is prescribed }\end{array}$ & $24 / 52(46.2)$ & $8 / 64(12.5)$ & $0.48 \mathrm{I}$ & 33.7 \\
\hline $\begin{array}{l}\text { d. Providing of spoken information regarding oxygen therapy in } \\
\text { patients in which home oxygen is prescribed }\end{array}$ & $25 / 50(50.0)$ & $16 / 62(25.8)$ & 0.241 & 24.2 \\
\hline $\begin{array}{l}\text { e. Providing of a leaflet with explanation regarding oxygen therapy } \\
\text { in patients in which home oxygen is prescribed }\end{array}$ & $18 / 50(36.0)$ & $12 / 62(19.4)$ & 0.219 & 16.6 \\
\hline $\begin{array}{l}\text { 15. Performance of revalidation tests during the past year (inclusive } \\
\text { current hospitalization) }\end{array}$ & $20 / 174(11.5)$ & $13 / 168(7.7)$ & 0.493 & 3.8 \\
\hline 16. Referral to pulmonary revalidation during the past year & $76 / 174(43.7)$ & $52 / 168(31.0)$ & 0.192 & 12.7 \\
\hline 17. Nutritional assessment (BMI) & $111 / 174(63.8)$ & $30 / 168(17.9)$ & 0.002 & 45.9 \\
\hline 18. Nutritional management in patients with underweight & $10 / 57(17.5)$ & $3 / 38(7.9)$ & 0.411 & 9.6 \\
\hline a. Referral to dietician in patients with underweight & $27 / 57(47.4)$ & $5 / 38(13.2)$ & 0.015 & 34.2 \\
\hline $\begin{array}{l}\text { b. Providing of nutritional advice in patients with } \\
\text { underweight }\end{array}$ & $21 / 57(36.8)$ & $4 / 38(10.5)$ & 0.040 & 26.3 \\
\hline c. Providing of nutritional supplement in patients with underweight & $21 / 57(36.8)$ & $8 / 38(21.1)$ & 0.354 & 15.7 \\
\hline 19. Nutritional management in patients with overweight & $10 / 28(35.7)$ & $4 / 23(17.4)$ & 0.443 & 18.3 \\
\hline a. Referral to dietician in patients with overweight & $16 / 28(57.1)$ & $5 / 23(21.7)$ & 0.051 & 35.4 \\
\hline $\begin{array}{l}\text { b. Providing of advice regarding weight loss in patients with } \\
\text { overweight }\end{array}$ & $14 / 28(50.0)$ & $6 / 23(26.1)$ & 0.372 & 23.9 \\
\hline 20. Patient received influenza vaccination within the past year & $113 / 174(64.9)$ & $97 / 168(57.7)$ & 0.991 & 7.2 \\
\hline 21. Patient received pneumococcal vaccination within the past 5 years & $66 / 174(37.9)$ & $85 / 168(50.6)$ & 0.076 & -12.7 \\
\hline $\begin{array}{l}\text { 22. ABG measurement I or } 2 \text { days prior to discharge in patients } \\
\text { hypoxemic during a COPD exacerbation }\end{array}$ & $22 / 51(43.1)$ & $20 / 46(43.5)$ & 0.921 & -0.4 \\
\hline $\begin{array}{l}\text { 23. Prescription of home oxygen therapy in patients with hypoxemia at } \\
\text { discharge }\end{array}$ & $3 / 5(60.0)$ & $4 / 15(26.7)$ & 0.566 & 33.3 \\
\hline $\begin{array}{l}\text { a. Proportion of patients in which home oxygen is prescribed in } \\
\text { patients who remain hypoxemic at discharge }\end{array}$ & $3 / 5(60.0)$ & $7 / 15(46.7)$ & 0.879 & 13.3 \\
\hline $\begin{array}{l}\text { b. Proportion of patients with indication for home oxygen therapy, } \\
\text { in which home oxygen therapy was prescribed for at least } \\
16 \text { hours a day in patients who remain hypoxemic at discharge }\end{array}$ & $3 / 5(60.0)$ & $4 / 15(26.7)$ & 0.566 & 33.3 \\
\hline
\end{tabular}




\begin{tabular}{|c|c|c|c|c|}
\hline & $\begin{array}{l}\text { Intervention group, } \\
\mathrm{n} / \mathrm{N}(\%)\end{array}$ & $\begin{array}{l}\text { Control group, } \\
n / N(\%)\end{array}$ & $P$-value & $\begin{array}{l}\text { Difference between } \\
\text { intervention and } \\
\text { control group }^{\mathrm{a}}\end{array}$ \\
\hline 24. Adequate discharge management & $5 \mathrm{I} / \mathrm{I} 74(29.3)$ & $26 / 168(15.5)$ & 0.330 & 13.8 \\
\hline a. Assessment of residence during current hospitalization & $169 / 174(97.1)$ & $151 / 168(89.9)$ & 0.862 & 7.2 \\
\hline b. Assessment of residential status within 3 days at admission & $151 / 169(89.3)$ & $|29 /| 5 \mid(85.4)$ & 0.940 & 3.9 \\
\hline c. Assessment of living status during current hospitalization & $150 / 174(86.2)$ & 149/168 (88.7) & 0.228 & -2.5 \\
\hline d. Assessment of living status within 3 days of admission & $122 / 150(8 \mid .3)$ & I28/I49 (85.9) & 0.289 & -4.6 \\
\hline e. Assessment of social status during current hospitalization & I53/I74 (87.9) & $145 / 168(86.3)$ & 0.501 & 1.6 \\
\hline f. Assessment of social support within 3 days & $115 / 153(75.2)$ & I24/I45 (85.5) & 0.199 & -10.3 \\
\hline g. Providing of spoken explanation about home medication & $88 / 174(50.6)$ & $78 / 167(46.7)$ & 0.855 & 3.9 \\
\hline $\begin{array}{l}\text { h. Providing of discharge letter with explanation about further } \\
\text { medication after discharge }\end{array}$ & $100 / 174(57.5)$ & $83 / 167(49.7)$ & 0.473 & 7.8 \\
\hline i. Providing of letter with information about follow-up appointment & $91 / 174(52.3)$ & $63 / 167(37.7)$ & 0.276 & 14.6 \\
\hline $\begin{array}{l}\text { j. Planning of follow-up appointment at 4-6 weeks after } \\
\text { discharge }\end{array}$ & 15 I/ $74(86.8)$ & $95 / 167(56.9)$ & $<0.001$ & 29.9 \\
\hline k. Availability of letter for general practitioner in medical record & 17|//74 (98.3) & $161 / 168(95.8)$ & 0.314 & 2.5 \\
\hline I. Support arranged at discharge if needed & $25 / 32(78.1)$ & $10 / 23(43.5)$ & $<0.001$ & 34.6 \\
\hline Prescription of nebulizer at the day of discharge & $63 / 174(36.2)$ & $67 / 168(39.9)$ & 0.898 & -3.7 \\
\hline
\end{tabular}

Note: aResults in bold refer to significance.

Abbreviations: $\mathrm{ABG}$, arterial blood gas; $\mathrm{BMI}$, body mass index; $\mathrm{FEV}$, forced expiratory volume at I second; NC, not possible to calculate.

rehabilitation, feelings of anxiety, readmission and in-hospital mortality. Due to limited statistical analysis and weak study design, the internal validity of results is limited. ${ }^{12-15}$ Our study confirmed that the implementation of a $\mathrm{CP}$ significantly reduces the 30 days readmission rate..$^{32,33}$

Although the 30 days readmission rate was significantly lower in the intervention group, no differences were found for readmission rate at 6 months. The 6 months readmission was chosen as primary outcome, based on previous studies and expert opinion. However, during the study, it became clear that the outcomes at 30 days after discharge are highly associated with the in-hospital treatment, which was the focus of the $\mathrm{CP}$ intervention, while results at 6 months are mainly related to the nature of the disease and the quality of the outpatient and primary care. Therefore, for future studies on in-hospital CPs and readmission, it is recommendable to primarily focus on the 30 days readmission. ${ }^{2}$ Finally, according to our results, $\sim 6$ of every 100 COPD patients treated according to the $\mathrm{CP}$ avoid a readmission at 30 days (number needed to treat: 17.0). Worldwide, based on the data of the WHO, this would result in a potential reduction of $\sim 4$ million readmissions. ${ }^{34}$

With regard to process indicators, only 2 of these results were statistically significant, and by implementing a $\mathrm{CP}$, the mean adherence to the guidelines was higher in the intervention group compared to the control group, though this difference was not significant. However, even non-significant results on process indicators may provide valid information regarding quality of care. Indeed, existing clinical practice guidelines on management of COPD exacerbation, which are very congruent and continuously updated, recommend unambiguously that the evaluated processes should be performed in every patient who is hospitalized for COPD exacerbation, regardless of patient characteristics or contextual factors. It is important to notice that, despite large improvements on process indicators after $\mathrm{CP}$ implementation, a considerable number of processes, especially with regard to non-pharmacological management, remained suboptimal performed. The non-pharmacological management contains some key interventions regarding education concerning smoking cessation, inhaler therapy and home oxygen therapy. Guidelines recommend more education as education is seen as an important part in the treatment of patients with a COPD exacerbation. ${ }^{2}$ After implementing the $\mathrm{CP}$, performance of education regarding inhaler therapy was significantly improved $(9.1 \%$ in the control group and 35.3\% in the intervention group; Table 4$)$. Although only $35.3 \%$ of the patients received education regarding inhaler therapy, while the other process indicators regarding education were even performed lower. So the care for patients with a COPD exacerbation is suboptimal performed, and therefore, continuous quality improvement will be needed in order to further optimize the care process for in-hospital management of COPD exacerbation and to enhance sustainability of the improved results. ${ }^{35,36}$ In addition, it is recommended that the team reconsiders the content of the pathway every 6 months. ${ }^{21}$ For instance, at the 
beginning of 2014, the evidence concerning optimal duration of glucocorticoid therapy for acute exacerbations of COPD was changed. ${ }^{2,37}$

Follow-up research is needed to understand why and under which circumstances CPs work in terms of which active components in CPs underlie their effect and what is the role of contextual factors and multidisciplinary teamwork. Furthermore, an economic evaluation should be included to evaluate whether CPs also impact efficiency of care. Finally, in the context of the rising prevalence of COPD, CPs should also include transmural management and community-based treatment of COPD. ${ }^{2}$

\section{Conclusion}

The implementation of this in-hospital CP for COPD exacerbation significantly reduced the 30 days readmission rate. This first international cluster randomized trial on CPs shows that the evidence-based key interventions are better performed after implementation of a CP compared to usual care. Additional studies are needed to understand how CPs are working, what their effect is on long term and how they affect the organization of care for different patient groups.

\section{Acknowledgments}

Cathy Lodewijckx is the joint first author with Kris Vanhaecht. We thank all professionals in the participating hospital wards who were involved in the implementation of the $\mathrm{CP}$ and the data collection. We thank R Veloso Mendes, P Boto, M Panella, F Leigheb, K Vanhaecht, W Sermeus, $\mathrm{S}$ Deneckere and C Lodewijckx for the coordination of the study in their country.

\section{Disclosure}

European Pathway Association obtained an unrestricted education grant from Pfizer SA, and this study was partially funded by Clinical Research Fund of University Hospitals Leuven. The funders had no role in the design, data collection, analysis, interpretation of data, writing of the report or decision to submit the report for publication. The autonomy of E-P-A and all involved academic institutions with regard to scientific independence and intellectual property on the methodology was guaranteed. MD has been part of Advisory Board for AstraZeneca, Boehringer-Pfizer, GSK, Nycomed, Novartis, Altana and Dompé. He has performed consulting work for Boehringer-Pfizer, GSK and Novartis. He also received lecture fees from these companies. All of the above amounted to $<10,000$ euro per annum. He received a research grant of 45,000 euro/year from AstraZeneca and 25,000 euro/ year from GSK. KV, WS and MP are board members of European Pathway Association. KV, CL, WS, SD, FL, PB, SK, DS and MP report no other conflicts of interest in this work.

\section{References}

1. Duman D, Aksoy E, Agca MC, et al. The utility of inflammatory markers to predict readmissions and mortality in COPD cases with or without eosinophilia. Int J Chron Obstruct Pulmon Dis. 2015;10: 2469-2478.

2. Global Initiative for Chronic Obstructive Lung Disesase. Global Strategy for Diagnosis, Management, and Prevention of COPD; 2015. Available from: http://www.goldcopd.it/materiale/2015/GOLD_Report_2015.pdf. Accessed February 11, 2015.

3. Izquierdo JL, Barcina C, Jimenez J, Muñoz M, Leal M. Study of the burden on patients with chronic obstructive pulmonary disease. Int $J$ Clin Pract. 2009;63(1):87-97.

4. Wedzicha JA, Seemungal TA. COPD exacerbations: defining their cause and prevention. Lancet. 2007;370(9589):786-796.

5. Map of Medicine (MOM) [homepage on the Internet]. Specialist management of acute exacerbation. Available from: http://mapofmedicine. com. Accessed November 2014.

6. Lodewijckx C, Sermeus W, Vanhaecht K, et al. Inhospital management of COPD exacerbations: a systematic review of the literature with regard to adherence to international guidelines. J Eval Clin Pract. 2009;15(6): 1101-1110.

7. Roberts CM, Lopez-Campos JL, Pozo-Rodriguez F, Hartl S; European COPD Audit Team. European hospital adherence to GOLD recommendations for chronic obstructive pulmonary disease (COPD) exacerbation admissions. Thorax. 2013;68:1169-1171.

8. Pearson SD, Goulart-Fisher D, Lee TH. Critical pathways as a strategy for improving care: problems and potential. Ann Intern Med. 1995;123: 941-948.

9. Rotter T, Kinsman L, James E, et al. Clinical pathways: effects on professional practice, patient outcomes, length of stay and hospital costs. Cochrane Database Syst Rev. 2010;3(9913):CD006632.

10. Costantini M, Romoli V, Leo SD, et al. Liverpool care pathway for patients with cancer in hospital: a cluster randomised trial. Lancet. 2014;383:226-237.

11. Vanhaecht K, Sermeus W, Peers J, et al. The impact of care pathways for exacerbation of chronic obstructive pulmonary disease: rationale and design of a cluster randomized controlled trial. Trials. 2010;11:111.

12. Lodewijckx C, Sermeus W, Panella M, et al. Impact of care pathways for in-hospital management of COPD exacerbation: a systematic review. Int J Nurs Stud. 2011;48:1445-1456.

13. Ban A, Ismail A, Harun R, Abdul Rahman A, Sulung S, Syed Mohamed A. Impact of clinical pathway on clinical outcomes in the management of COPD exacerbation. BMC Pulm Med. 2012;12:27.

14. Nishimura K, Yasui M, Nishimura T, Oga T. Clinical pathway for acute exacerbations of chronic obstructive pulmonary disease: method development and five years of experience. Int J Chron Obstruct Pulmon Dis. 2011;6:365-372.

15. Abd-Elwanees A, El-Soussi A, Othman S, Ali R. Effect of implementing clinical pathway on the clinical outcomes of patients with acute exacerbations of chronic obstructive pulmonary disease. Int J Nurs Sci. 2014;4:1-10.

16. Craig P, Dieppe P, Macintyre S, et al. Developing and evaluating complex interventions: the new Medical Research Council guidance. BMJ. 2008;337:a1655.

17. Patsopoulos NA. A pragmatic view on pragmatic trials. Dialogues Clin Neurosci. 2011;13(2):217-224.

18. Hosker H, Anstey K, Lowe D, Pearson M, Roberts CM. Variability in the organisation and management of hospital care for COPD exacerbations in the UK. Respir Med. 2007;101:754-761. 
19. Roberts CM, Barnes S, Lowe D, et al. Evidence for a link between mortality in acute COPD and hospital type and resources. Thorax. 2003; 58:947-949.

20. Lodewijckx C, Decramer M, Sermeus W, Panella M, Deneckere S, Vanhaecht K. Eight-step method to build the clinical content of an evidence-based care pathway: the case for COPD exacerbation. Trials. 2012;13:229

21. Vanhaecht K, Van Gerven E, Deneckere S, et al. The 7-phase method to design, implement and evaluate care pathways. Int J Per Centered Med. 2012;2:341-351.

22. Deneckere S, Euwema M, Lodewijckx C, et al. Better interprofessional teamwork, higher level of organized care, and lower risk of burnout in acute health care teams using care pathways: a cluster randomized controlled trial. Med Care. 2013;51(1):99-107.

23. Panella M, Marchisio S, Demarchi ML, Manzoli L, Di Stanislao F. Reduced in-hospital mortality for heart failure with clinical pathways: the results of a cluster randomised controlled trial. Qual Saf Health Care. 2009;18:369-373.

24. Campbell MK, Mollison J, Grimshaw JM. Cluster trials in implementation research: estimation of intracluster correlation coefficients and sample size. Stat Med. 2001;20(3):391-399.

25. Almagro P, Barreiro B, Ochoa de Echaguen A, et al. Risk factors for hospital readmission in patients with chronic obstructive pulmonary disease. Respiration. 2006;73(6):311-317.

26. Bratzler DW, Oehlert WH, McAdams LM, Leon J, Jiang H, Piatt D. Management of acute exacerbations of chronic obstructive pulmonary disease in the elderly: physician practices in the community hospital setting. J Okla State Med Assoc. 2004;97:227-232.

27. Cunningham S, Logan C, Lockerbie L, Dunn MJ, McMurray A, Prescott RJ. Effect of an integrated care pathway on acute asthma/wheeze in children attending hospital: cluster randomized trial. J Pediatr. 2008; 152(3):315-320.

28. Kinsman LD, Rotter T, Willis J, Snow PC, Buykx P, Humphreys JS. Do clinical pathways enhance access to evidence-based acute myocardial infarction treatment in rural emergency departments? Aust J Rural Health. 2012;20(2):59-66.
29. Panella M, Marchisio S, Barbieri A, Di Stanislao F. A cluster randomized trial to assess the impact of clinical pathways for patients with stroke: rationale and design of the Clinical Pathways for Effective and Appropriate Care Study [NCT00673491]. BMC Health Serv Res. 2008;8:223.

30. De Luca A, Toni D, Lauria L, et al. An emergency clinical pathway for stroke patients - results of a cluster randomised trial (isrctn41456865). BMC Health Serv Res. 2009;9:14.

31. Medical Research Council (MRC). Cluster Randomised Trials: Methodological and Ethical Considerations; 2002. Available from: http://www.cebma.org/wp-content/uploads/Cluster-randomised-trialsMethodological-and-ethical-considerations.pdf. Accessed April 2015.

32. Santamaria N, Conners A, Osteraas J, Ham J, Boodram B. A prospective cohort study of the effectiveness of clinical pathways for the in-patient management of acute exacerbation of chronic obstructive pulmonary disease (COPD). Collegian. 2004;11:12-16.

33. McManus TE, Marley A, Kidney JC. The Mater Hospital multiprofessional care pathway for acute exacerbations of chronic obstructive pulmonary disease. J Integr Care Pathways. 2005;9:32-36.

34. WHO [webpage on the Internet]. Chronic obstructive pulmonary disease (COPD): facts. Available from: http://www.who.int/respiratory/copd/en/. Accessed February 2015.

35. Shortell SM, Bennett CL, Byck GR. Assessing the impact of continuous quality improvement on clinical practice: what it will take to accelerate progress. Milbank Q. 1998;76:593-624.

36. Wiltsey SS, Kimberly J, Cook N, Calloway A, Castsro F, Charns M. The sustainability of new programs and innovations: a review of the empirical literature and recommendations for future research. Implement Sci. 2012;7:17.

37. Vogelmeier CF, Vestbo J, Hurd SS, et al. Changes in GOLD: today and tomorrow. Lancet Respir Med. 2015;3:424-426. 


\title{
Supplementary material
}

Table SI List of participating hospitals that gave ethical approval for this study

\author{
ASL AT \\ AO Mauriziano di Torino \\ AOU Maggiore della Carità di Novara ward I \\ AOU Maggiore della Carità di Novara ward 2 \\ AOU San Luigi di Orbassano \\ ASL AL \\ ASL TOI \\ ASL VC \\ AZ Sint - Augustinus Wilrijk \\ AZ Sint Elizabeth Zottegem \\ AZ Sint-Blasius Dendermonde \\ AZ Sint-Jan AV Brugge \\ Centro Hospitalar Lisboa Central \\ Heilig Hart ziekenhuis Roeselare - Menen campus Menen \\ Heilig Hart ziekenhuis Roeselare Menen campus Roeselare \\ Hospital Distrital de Faro \\ Mariaziekenhuis Overpelt \\ RZ Jan Yperman leper \\ Sint-Elisabeth Ziekenhuis Turnhout \\ Sint-Vincentius ziekenhuis, Antwerpen \\ Virga Jesse Ziekenhuis Hasselt \\ ZiekenhuisNetwerk Antwerpen campus Jan Palfijn
}

Abbreviations: ASL AT, Azienda Sanitaria Locale di Asti; AO, Azienda Ospedaliera; AOU, Azienda Ospedaliero Universitaria; ASL AL, Azienda Sanitaria Locale della provincia di Alessandria; ASL VC, Azienda Sanitaria Locale di Vercelli; ASL TOI, Azienda Sanitaria Locale Torino; AZ, Algemeen ziekenhuis; RZ, Regionaal ziekenhuis.

\section{Publish your work in this journal}

The International Journal of COPD is an international, peer-reviewed journal of therapeutics and pharmacology focusing on concise rapid reporting of clinical studies and reviews in COPD. Special focus is given to the pathophysiological processes underlying the disease, intervention programs, patient focused education, and self management protocols.

\section{Dovepress}

This journal is indexed on PubMed Central, MedLine and CAS. The manuscript management system is completely online and includes a very quick and fair peer-review system, which is all easy to use. Visit $\mathrm{http}: / /$ www.dovepress.com/testimonials.php to read real quotes from published authors. 